\title{
Maximum supercurrent in two Josephson-junction stacks: Theory and experiment
}

\author{
Carapella, G; Costabile, G; Sakai, S; Pedersen, Niels Falsig
}

Published in:

Physical Review B

Link to article, DOI:

10.1103/PhysRevB.58.6497

Publication date:

1998

Document Version

Publisher's PDF, also known as Version of record

Link back to DTU Orbit

Citation (APA):

Carapella, G., Costabile, G., Sakai, S., \& Pedersen, N. F. (1998). Maximum supercurrent in two Josephsonjunction stacks: Theory and experiment. Physical Review B, 58(10), 6497-6505.

https://doi.org/10.1103/PhysRevB.58.6497

\section{General rights}

Copyright and moral rights for the publications made accessible in the public portal are retained by the authors and/or other copyright owners and it is a condition of accessing publications that users recognise and abide by the legal requirements associated with these rights.

- Users may download and print one copy of any publication from the public portal for the purpose of private study or research.

- You may not further distribute the material or use it for any profit-making activity or commercial gain

- You may freely distribute the URL identifying the publication in the public portal

If you believe that this document breaches copyright please contact us providing details, and we will remove access to the work immediately and investigate your claim. 


\title{
Maximum supercurrent in two Josephson-junction stacks: Theory and experiment
}

\author{
G. Carapella and G. Costabile \\ Unità INFM and Dipartimento di Fisica, Università di Salerno, I-84081 Baronissi, Italy \\ S. Sakai \\ Electrotechnical Laboratory, 1-1-4 Umezono, Tsukuba-shi, Ibaraki 305, Japan \\ N. F. Pedersen \\ Department of Physics, B309, The Technical University of Denmark, DK-2800 Lyngby, Denmark
}

(Received 19 March 1998)

\begin{abstract}
The interaction between two long Josephson junctions in a stack is investigated experimentally in the absence of applied magnetic field. Mutual interaction is observed when both junctions or only one junction in the stack is in the zero voltage state. To account for the observed phenomena we propose a model that takes into account the nonuniform self-fields generated by the bias currents. [S0163-1829(98)02834-3]
\end{abstract}

\section{INTRODUCTION}

In recent years the double-barrier stacked structure in which two Josephson junctions have in common the middle electrode has received much attention. This structure is regarded as the basic block of some layered high- $T_{c}$ superconducting materials (Bi-Sr-Ca-Cu-O family), so stimulating a strong theoretical interest. However, for the variety of new physical phenomena exhibited by the double-barrier stack, it is also regarded as an interesting device with potential application in superconducting electronics, principally as a threeterminal device. In fact, in the three-terminal configuration the device is shown to support a variety of flux motion synchronization phenomena, useful to the improvement of the existing fluxon oscillators. Moreover, the nonequilibrium phenomena that can occur in the structure might also be considered for applications. For example, by polarizing one of the two junctions of the stack (generator junction) at a voltage larger than the gap sum voltage we can inject quasiparticles in the intermediate (common) electrode, causing a quasiparticle excess in the same electrode. Some of these excess quasiparticles can tunnel the barrier of the second junction (detector junction) with a modification of the quasiparticle curve (rise of the subgap current and depression of the gap voltage). Such a behavior could be attractive for the implementation of a transistorlike device. So far, there are some open questions concerning the dominant mechanism of interlayer coupling in this system. Nonequilibrium injection of quasiparticles and suppression of the order parameter might play an important role in the case of a very thin middle electrode (thinner than the coherence length $\xi$ ), very high Josephson current density, and very small junctions. The inductive (or magnetic) coupling, which is due to the screening currents in the common electrode when its thickness is about equal or smaller than the London penetration depth $\lambda_{L}$, is regarded as the dominant one, especially when the physical dimensions of the junctions are larger than the Josephson penetration length $\lambda_{J}$. In fact such a coupling, which has been elegantly formalized in a model ${ }^{1}$ for the multilayered structures, accounts for many dynamical phenomena in long stacked junctions, including synchronization of fluxon motion. If we remain in the limit of the inductive coupling, we expect that for a full description of a three-terminal device we have to take care of the geometrical factors, i.e., we must include in a realistic model the effects due to the boundary conditions that add up to the bulk magnetic interaction. In other words, due the magnetic nature of the coupling, the self-fields generated by the currents feeding the device must be taken into account.

The above considerations concerning the dominant mechanism for the interaction are suggested by the experimental evidence that the maximum supercurrent in one junction of the stack depends on the value of the bias current and of the voltage across the other junction. In this paper we report on the measurements of the maximum supercurrent in three terminal devices consisting of two long stacked Josephson junctions with a double overlap geometry. We also describe a theoretical procedure to include the geometrical effects in three terminal devices and compare the experimental data with the prediction of the model. The paper is organized as follows. In Sec. I we present the general two-dimensional inductive model for a two junction stack and its application to the special case of the double overlap geometry. In the one-dimensional approximation for this geometry we show how the self-fields generated by the bias currents translate into the model of coupled sine-Gordon equations. The realistic situation of nonuniform self-fields is considered as well as the classic uniform approximation. In Sec. II we present experimental results demonstrating static interactions between junctions in the stack. We discuss the possible mechanisms that could be causing the observed interactions and we show that the dominant one is the nonuniform current distribution of the bias currents. Experimental results are then compared with the numerical results of the proposed model.

\section{TWO-DIMENSIONAL INDUCTIVE MODEL FOR A TWO JUNCTION STACK}

We are considering the stack configuration and orientation shown in Fig. 1; the bottom and the top junctions will be 


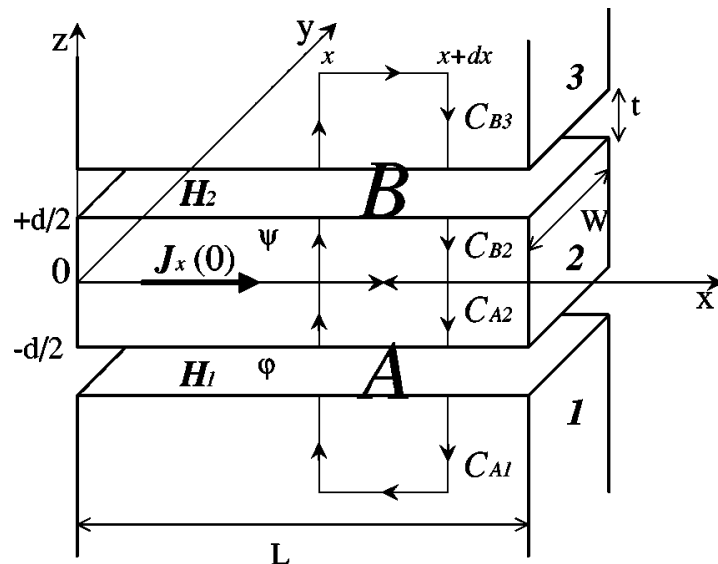

FIG. 1. Sketch of the stack geometry.

named, respectively, $A$ and $B$. For the sake of simplicity we shall assume that the external electrodes are much thicker than the London penetration depth $\lambda_{L}$; this assumption, of course, does not apply to the middle electrode. Moreover, we are also assuming (but this assumption can be easily relieved) that the two junctions are identical. The basic steps of this derivation closely follow the classical approach for single junctions (see, e.g., Ref. 2). We start from the Ginzburg-Landau equation

$$
\nabla \theta=\frac{2 \pi}{\Phi_{0}}\left(\mathbf{A}+\frac{4 \pi}{c} \lambda_{L}^{2} \mathbf{J}\right)
$$

that we shall integrate along the path shown in the figure. We first focus the attention on the junction defined by the electrodes 2 and 1 in the figure. Integrating Eq. (1) along the path $C_{A 2}$ in the middle electrode to the barrier we obtain

$$
\theta_{2}(x+d x)-\theta_{2}(x)=\frac{2 \pi}{\Phi_{0}}\left(\int_{C_{A 2}} \mathbf{A} \cdot d \mathbf{l}+\frac{4 \pi}{c} \lambda_{L}^{2} J_{x}(0) d x\right) .
$$

Integrating Eq. (1) along the path $C_{A 1}$ in the bottom electrode we have, instead,

$$
-\theta_{1}(x+d x)+\theta_{1}(x)=\frac{2 \pi}{\Phi_{0}}\left(\int_{C_{A 1}} \mathbf{A} \cdot d \mathbf{l}\right)
$$

as we may choose the side of the path parallel to the $x$ axis to make negligible $J_{x}$. Adding these two equations and defining $\varphi \equiv \theta_{2}-\theta_{1}$ we get

$$
\varphi(x+d x)-\varphi(x)=\frac{2 \pi}{\Phi_{0}}\left(\oint \mathbf{A} \cdot d \mathbf{l}+\frac{4 \pi}{c} \lambda_{L}^{2} J_{x}(0) d x\right),
$$

where the barrier thickness has been neglected. The last equation can be rewritten as

$$
d \varphi=\frac{2 \pi}{\Phi_{0}}\left(\Phi_{A}\left(H_{y}\right)+\frac{4 \pi}{c} \lambda_{L}^{2} J_{x}(0) d x\right),
$$

where $\Phi_{A}\left(H_{y}\right)$ is the flux of the $y$ component of the magnetic field threading the integration path for junction $A$.

Following the same procedure, we will get an analogous equation for the other junction:

$$
d \psi=\frac{2 \pi}{\Phi_{0}}\left(\Phi_{B}\left(H_{y}\right)-\frac{4 \pi}{c} \lambda_{L}^{2} J_{x}(0) d x\right),
$$

where $\psi \equiv \theta_{3}-\theta_{2}$.

Dividing the last two equations by $d x$ we may write

$$
\begin{aligned}
& \varphi_{x}=\frac{2 \pi}{\Phi_{0}}\left(\frac{\Phi_{A}\left(H_{y}\right)}{d x}+\frac{4 \pi}{c} \lambda_{L}^{2} J_{x}(0)\right), \\
& \psi_{x}=\frac{2 \pi}{\Phi_{0}}\left(\frac{\Phi_{B}\left(H_{y}\right)}{d x}-\frac{4 \pi}{c} \lambda_{L}^{2} J_{x}(0)\right) .
\end{aligned}
$$

For a generic orientation of the magnetic field in the plane of junctions, we have to take into account the $x$ component of the field. Applying the same procedure to a path in the $z-y$ plane and taking care of the fact that the sign of the integration path is determined by sign of $H_{x}$, we find similar equations, but with opposite sign:

$$
\begin{gathered}
\varphi_{y}=-\frac{2 \pi}{\Phi_{0}}\left(\frac{\Phi_{A}\left(H_{x}\right)}{d y}+\frac{4 \pi}{c} \lambda_{L}^{2} J_{y}(0)\right), \\
\psi_{y}=-\frac{2 \pi}{\Phi_{0}}\left(\frac{\Phi_{B}\left(H_{x}\right)}{d y}-\frac{4 \pi}{c} \lambda_{L}^{2} J_{y}(0)\right) .
\end{gathered}
$$

To evaluate terms in Eqs. (2) and (3) we need an expression for $\mathbf{H}(z)$ and for $\mathbf{J}(z)$.

An expression for the field $\mathbf{H}(z)=\left[H_{x}(z), H_{y}(z), 0\right]$ can be obtained from London equation

$$
\frac{\partial^{2} \mathbf{H}}{\partial z^{2}}=\frac{1}{\lambda_{L}^{2}} \mathbf{H}
$$

with the proper boundary conditions (b.c.). If $\mathbf{H}_{\mathbf{1}}$ and $\mathbf{H}_{\mathbf{2}}$ are the magnetic fields in the junction barriers, the solutions of Eq. (4) will decrease exponentially in electrodes 1 and 3, while in the middle electrode we get

$$
\mathbf{H}(z)=\frac{\mathbf{H}_{\mathbf{1}}+\mathbf{H}_{\mathbf{2}}}{2} \frac{\cosh \left(z / \lambda_{L}\right)}{\cosh \left(d / 2 \lambda_{L}\right)}+\frac{\mathbf{H}_{\mathbf{2}}-\mathbf{H}_{\mathbf{1}}}{2} \frac{\sinh \left(z / \lambda_{L}\right)}{\sinh \left(d / 2 \lambda_{L}\right)} .
$$

From this equation we may now also calculate the screening current $J_{x}(0)$ in the middle electrode (for the other electrodes we already know that it will be exponentially decreasing as we move from the internal surface to the outside). From Maxwell equation

$$
\nabla \times \mathbf{H}=\frac{4 \pi}{c} \mathbf{J}
$$

we obtain

$$
\begin{aligned}
& J_{x}(0)=-\frac{c}{4 \pi \lambda_{L}}\left(\frac{H_{2 y}-H_{1 y}}{2} \frac{1}{\sinh \left(d / 2 \lambda_{L}\right)}\right), \\
& J_{y}(0)=-\frac{c}{4 \pi \lambda_{L}}\left(\frac{H_{2 x}-H_{1 x}}{2} \frac{1}{\sinh \left(d / 2 \lambda_{L}\right)}\right) .
\end{aligned}
$$

From this, we may write our fundamental equations (2) and (3) as 


$$
\begin{gathered}
\varphi_{x}=\frac{2 \pi}{\Phi_{0}} d^{\prime}\left(H_{1 y}+\varepsilon H_{2 y}\right), \\
\psi_{x}=\frac{2 \pi}{\Phi_{0}} d^{\prime}\left(H_{2 y}+\varepsilon H_{1 y}\right), \\
\varphi_{y}=-\frac{2 \pi}{\Phi_{0}} d^{\prime}\left(H_{1 x}+\varepsilon H_{2 x}\right), \\
\psi_{y}=-\frac{2 \pi}{\Phi_{0}} d^{\prime}\left(H_{2 x}+\varepsilon H_{1 x}\right),
\end{gathered}
$$

where we have defined

$$
d^{\prime} \equiv t+\lambda_{L}+\lambda_{L} \operatorname{coth}\left(\frac{d}{\lambda_{L}}\right)
$$

and

$$
\varepsilon \equiv-\frac{\lambda_{L}}{\sinh \left(d / \lambda_{L}\right)} \frac{1}{\left[t+\lambda_{L}+\lambda_{L} \operatorname{coth}\left(d / \lambda_{L}\right)\right]} .
$$

If $d / \lambda_{L} \rightarrow \infty$ (very thick intermediate electrode), $\varepsilon$ $\rightarrow 0, d^{\prime}=t+2 \lambda_{L}$, as we should expect in the limit of noninteracting junctions. In a more compact form, the obtained phase-field relations are

$$
\begin{aligned}
& \nabla_{x, y} \varphi=\frac{2 \pi}{\Phi_{0}} d^{\prime}\left(\mathbf{H}_{\mathbf{1}}+\varepsilon \mathbf{H}_{2}\right) \times \hat{z} \\
& \nabla_{x, y} \psi=\frac{2 \pi}{\Phi_{0}} d^{\prime}\left(\mathbf{H}_{2}+\varepsilon \mathbf{H}_{1}\right) \times \hat{z} .
\end{aligned}
$$

We have now to combine the Maxwell equations with the Josephson relations. The $z$ component of Eq. (5) in the two barriers is written as

$$
\begin{aligned}
& \frac{\partial H_{1 y}}{\partial x}-\frac{\partial H_{1 x}}{\partial y}=\frac{4 \pi}{c} J_{1 z}, \\
& \frac{\partial H_{2 y}}{\partial x}-\frac{\partial H_{2 x}}{\partial y}=\frac{4 \pi}{c} J_{2 z},
\end{aligned}
$$

where

$$
\begin{gathered}
J_{1 z}=J_{0} \sin (\varphi)+C \frac{\partial V_{1}}{\partial t}+\frac{V_{1}}{R}, \\
J_{2 z}=J_{0} \sin (\psi)+C \frac{\partial V_{2}}{\partial t}+\frac{V_{2}}{R}, \\
C=\frac{\epsilon_{r}}{4 \pi t} ; \quad V_{1}=\frac{\hbar}{2 e} \varphi_{t} ; \quad V_{2}=\frac{\hbar}{2 e} \psi_{t} .
\end{gathered}
$$

Substituting into Eqs. (9), taking into account Eqs. (8), we obtain the two equations

$$
\varphi_{x x}+\varphi_{y y}-\frac{1}{\bar{c}^{2}} \varphi_{t t}=\frac{1}{\lambda_{j}^{2}} \sin (\varphi)+\bar{\alpha} \varphi_{t}+\varepsilon\left(\psi_{x x}+\psi_{y y}\right),
$$

$$
\psi_{x x}+\psi_{y y}-\frac{1}{\bar{c}^{2}} \psi_{t t}=\frac{1}{\lambda_{j}^{2}} \sin (\psi)+\bar{\alpha} \psi_{t}+\varepsilon\left(\varphi_{x x}+\varphi_{y y}\right)
$$

were we have introduced the Swihart velocity

$$
\bar{c}=c \sqrt{\frac{t}{\epsilon_{r} d^{\prime}\left(1-\varepsilon^{2}\right)}},
$$

the Josephson penetration depth

$$
\lambda_{J}=\sqrt{\frac{\hbar c^{2}}{8 \pi e d^{\prime}\left(1-\varepsilon^{2}\right) J_{0}}},
$$

and the (dimensional) loss parameter

$$
\bar{\alpha}=\frac{d^{\prime}\left(1-\varepsilon^{2}\right) 4 \pi}{c^{2} R} .
$$

So far no direct reference to the geometry of the stack has been necessary. Geometric considerations enter, of course, through the boundary conditions. The appropriate b.c. (Neumann type) require specification of normals components of $\nabla \varphi$ and $\nabla \psi$ at the junctions boundary $C_{1}$ and $C_{2}$. By Eqs. (8) we have

$$
\begin{aligned}
& \left.\nabla \varphi \cdot \widehat{\mathbf{n}_{1}}\right|_{C_{1}}=\frac{2 \pi}{\Phi_{0}} d^{\prime}\left(\mathbf{H}_{1}+\varepsilon \mathbf{H}_{2}\right) \times\left.\hat{z} \cdot \widehat{\mathbf{n}_{1}}\right|_{C_{1}}, \\
& \left.\nabla \psi \cdot \widehat{\mathbf{n}_{2}}\right|_{C_{2}}=\frac{2 \pi}{\Phi_{0}} d^{\prime}\left(\mathbf{H}_{2}+\varepsilon \mathbf{H}_{1}\right) \times\left.\hat{z} \cdot \widehat{\mathbf{n}_{2}}\right|_{C_{2}},
\end{aligned}
$$

where $\widehat{\mathbf{n}_{1}}$ and $\widehat{\mathbf{n}_{2}}$ are the unit normal vector of contours $C_{1}$ and $C_{2}$, respectively. In the special case of rectangular geometry $(0 \leqslant x \leqslant L ; 0 \leqslant y \leqslant W)$ these b.c. became

$$
\begin{gathered}
\left.\varphi_{x}\right|_{0, L}=\left.\frac{2 \pi}{\Phi_{0}} d^{\prime}\left(H_{1 y}+\varepsilon H_{2 y}\right)\right|_{0, L}, \\
\left.\psi_{x}\right|_{0, L}=\left.\frac{2 \pi}{\Phi_{0}} d^{\prime}\left(H_{2 y}+\varepsilon H_{1 y}\right)\right|_{0, L}, \\
\left.\varphi_{y}\right|_{0, W}=-\left.\frac{2 \pi}{\Phi_{0}} d^{\prime}\left(H_{1 x}+\varepsilon H_{2 x}\right)\right|_{0, W}, \\
\left.\psi_{y}\right|_{0, W}=-\left.\frac{2 \pi}{\Phi_{0}} d^{\prime}\left(H_{2 x}+\varepsilon H_{1 x}\right)\right|_{0, W}
\end{gathered}
$$

where the fields include both self-fields (generated by the bias currents) and externally applied fields.

\section{A. One-dimensional approximation for the double overlap geometry}

We will consider the special case of the one-dimensional approximation for the double overlap geometry (see Fig. 2), that has been extensively investigated experimentally and has demonstrated very rich dynamics, also without applied magnetic field. ${ }^{3,4}$ In this geometry the two junctions can be biased independently. To make our approximation to the one-dimensional case we will proceed as for the single junc- 


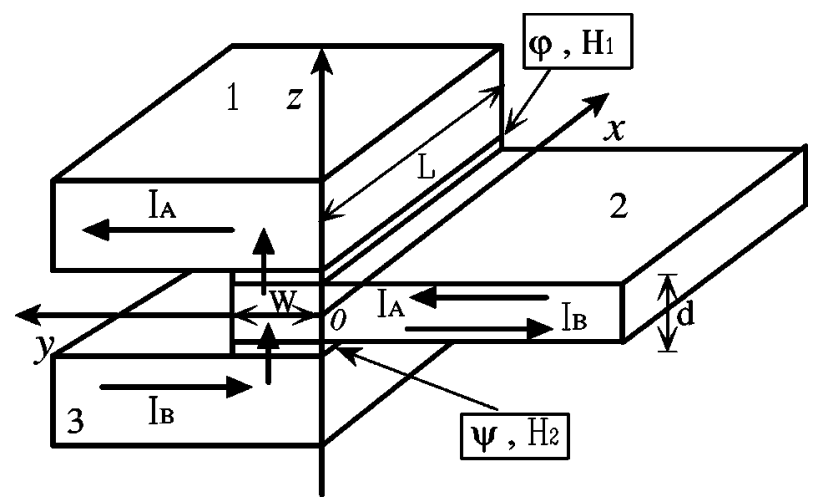

FIG. 2. Double overlap geometry.

tion. Since $W \ll \lambda_{j}$ we expect the phases and fields to depend very weakly (the phases at most quadratically ${ }^{5}$ and the field at most linearly) on $y$. To the first order in $W$, integrating Eqs. (10) along $y$, between $y=0$ and $y=W$, and using Eqs. (15) we have

$$
\begin{aligned}
& \varphi_{x x}-\frac{1}{\bar{c}^{2}} \varphi_{t t}=\frac{1}{\lambda_{j}^{2}} \sin (\varphi)+\bar{\alpha} \varphi_{t}+\varepsilon \psi_{x x}+\Gamma_{A}(x), \\
& \psi_{x x}-\frac{1}{\bar{c}^{2}} \psi_{t t}=\frac{1}{\lambda_{j}^{2}} \sin (\psi)+\bar{\alpha} \psi_{t}+\varepsilon \varphi_{x x}+\Gamma_{B}(x),
\end{aligned}
$$

where

$$
\begin{aligned}
& \Gamma_{A}(x)=\frac{c}{4 \pi \lambda_{J}^{2} J_{0} W}\left[H_{1 x}(x, W)-H_{1 x}(x, 0)\right], \\
& \Gamma_{B}(x)=\frac{c}{4 \pi \lambda_{J}^{2} J_{0} W}\left[H_{2 x}(x, W)-H_{2 x}(x, 0)\right] .
\end{aligned}
$$

As the bias currents flow in the $y$ direction (see Fig. 2), the magnetic field in this same direction will be negligible, so for this geometry self-fields do not enter in the boundary conditions of the one-dimensional approximation. Evaluation of bias-dependent terms $\Gamma_{A}$ and $\Gamma_{B}$ depends on the assumptions made for the currents distributions into the electrodes. Here we will consider the classical assumption of uniform current distribution and the case of nonuniform current distribution.

\section{Uniform current distribution}

If the bias current distribution into the elecrodes is assumed uniform, then the fields of interest are

$$
\begin{gathered}
H_{1 x}(0)=\frac{2 \pi}{c} \frac{1}{L}\left(I_{A}-I_{B}\right), \\
H_{1 x}(W)=-\frac{2 \pi}{c} \frac{1}{L}\left(I_{A}+I_{B}\right), \\
H_{2 x}(0)=\frac{2 \pi}{c} \frac{1}{L}\left(I_{B}-I_{A}\right),
\end{gathered}
$$

$$
H_{2 x}(W)=-\frac{2 \pi}{c} \frac{1}{L}\left(I_{A}+I_{B}\right) .
$$

From Eqs. (17) we have

$$
\begin{aligned}
& \Gamma_{A}(x)=-\frac{I_{A}}{L \lambda_{J}^{2} J_{0} W}=-\frac{1}{\lambda_{J}^{2}} \frac{I_{A}}{I_{0}} \equiv-\frac{1}{\lambda_{J}^{2}} \gamma_{A}, \\
& \Gamma_{B}(x)=-\frac{I_{B}}{L \lambda_{J}^{2} J_{0} W}=-\frac{1}{\lambda_{J}^{2}} \frac{I_{B}}{I_{0}} \equiv-\frac{1}{\lambda_{J}^{2}} \gamma_{B} .
\end{aligned}
$$

Inserting the last two equations in Eqs. (17), normalizing the lengths with respect to $\lambda_{j}$ and the time to the inverse of $\omega_{j}$ $=\bar{c} / \lambda_{j}$, we finally find

$$
\begin{gathered}
\varphi_{x x}-\varphi_{t t}=\sin (\varphi)+\alpha \varphi_{t}+\varepsilon \psi_{x x}-\gamma_{A}, \\
\psi_{x x}-\psi_{t t}=\sin (\psi)+\alpha \psi_{t}+\varepsilon \varphi_{x x}-\gamma_{B}, \\
\varphi_{x}(0)=\varphi_{x}(l)=\eta(1+\varepsilon), \\
\psi_{x}(0)=\psi_{x}(l)=\eta(1+\varepsilon),
\end{gathered}
$$

with $l=L / \lambda_{J}$ and $\alpha=(1 / R) \sqrt{\hbar /\left(2 e C J_{0}\right)}$. The $\eta$ term in the boundary conditions accounts for an external magnetic field (e.g., given by a coil) parallel to the $y$ direction, normalized with respect to $\lambda_{j}\left(1-\varepsilon^{2}\right)(4 \pi / c) J_{0}$.

\section{Nonuniform current distribution}

It is well known ${ }^{6,7}$ that the current distribution in a long and thin strip is highly nonuniform across its width $L$. In the past, this fact has been considered for explanation ${ }^{8-10}$ of deviation from ideal behavior of the single overlap junctions. In fact, these junctions consist of an interruption by an oxide layer of a long superconducting strip and, according to the shortening principle, ${ }^{11}$ the real current distribution is as the current distribution in the strip.

When the film thickness $d$ is comparable with the penetration depth $\lambda_{L}$ and the width $L$ is much greater than $\lambda_{L}$, so that $L d \gg \lambda_{L}^{2}$, a good approximation for the linear current density in an isolated long strip is: $6,7,12,13$

$$
J(x)=\frac{I}{\pi L} \frac{L}{\sqrt{x(L-x)}} .
$$

Near the edges of the strip the distribution (21) has an exponential correction that, extending over a length approximately equal to $\lambda_{L}$, prevents from singularities at $x=0$ and $x=L$ and sets a relation between the current density at the edges and that at the center ${ }^{13}$

$$
\frac{J(0)}{J(L / 2)}=\frac{1.165}{\lambda_{L}} \sqrt{\frac{L d}{a}},
$$

where $a$ is a constant near unity.

In our geometry (see Fig. 2) the distribution Eq. (21) is appropriate for the middle electrode. For the outer electrodes we also expect a nonuniform current distribution, but much less drastic. For the current component whose magnitude is the same in both electrodes but whose flow (relative to the $y$ direction) is opposite, we can expect a current distribution 
almost uniform because the separation between them is very small (typically of order of $d \approx \lambda_{L}$ ). From the geometrical symmetry, it may also be assumed that the current coming from the middle electrode is divided equally into the two outer electrodes. This gives rise to a current component in the outer electrodes having the same magnitude and polarity. When we express $I_{A}$ and $-I_{B}$ as $I_{A}=\left(I_{A}+I_{B}\right) / 2+\left(I_{A}\right.$ $\left.-I_{B}\right) / 2$ and $-I_{B}=-\left(I_{A}+I_{B}\right) / 2+\left(I_{A}-I_{B}\right) / 2$, their first and second terms correspond to the opposite and equal polarity components, respectively. For the calculation in $H_{1 x}(W)$ and $H_{2 x}(W)$, the contributions from the second terms in the two equations are canceled when summed. For what concern the first terms, we can assume a uniform current distribution as described above. By these considerations the fields of interest are

$$
\begin{gathered}
H_{1 x}(0)=\frac{2 \pi}{c} \frac{1}{L}\left(I_{A}-I_{B}\right) \frac{L}{\pi \sqrt{x(L-x)}}, \\
H_{1 x}(W)=-\frac{2 \pi}{c} \frac{1}{L}\left(I_{A}+I_{B}\right), \\
H_{2 x}(0)=\frac{2 \pi}{c} \frac{1}{L}\left(I_{B}-I_{A}\right) \frac{L}{\pi \sqrt{x(L-x)}}, \\
H_{2 x}(W)=-\frac{2 \pi}{c} \frac{1}{L}\left(I_{A}+I_{B}\right),
\end{gathered}
$$

that we can insert in Eqs. (17) to have

$$
\begin{aligned}
& \Gamma_{A}(x)=-\frac{1}{\lambda_{J}^{2}}\left(\frac{\gamma_{A}+\gamma_{B}}{2}+\frac{\gamma_{A}-\gamma_{B}}{2} \frac{L}{\pi \sqrt{x(L-x)}}\right), \\
& \Gamma_{B}(x)=-\frac{1}{\lambda_{J}^{2}}\left(\frac{\gamma_{A}+\gamma_{B}}{2}+\frac{\gamma_{B}-\gamma_{A}}{2} \frac{L}{\pi \sqrt{x(L-x)}}\right),
\end{aligned}
$$

where $\gamma_{A}=I_{A} / J_{0} W L, \gamma_{B}=I_{B} / J_{0} W L$. From this, by using Eqs. (16) and normalizing as in the uniform current case, we finally find

$$
\begin{gathered}
\varphi_{x x}-\varphi_{t t}=\sin (\varphi)+\alpha \varphi_{t}+\varepsilon \psi_{x x}-\eta_{A}(x), \\
\psi_{x x}-\psi_{t t}=\sin (\psi)+\alpha \psi_{t}+\varepsilon \varphi_{x x}-\eta_{B}(x), \\
\varphi_{x}(0)=\varphi_{x}(l)=\eta(1+\varepsilon), \\
\psi_{x}(0)=\psi_{x}(l)=\eta(1+\varepsilon),
\end{gathered}
$$

where

$$
\begin{aligned}
& \eta_{A}(x)=\frac{\gamma_{A}+\gamma_{B}}{2}+\frac{\gamma_{A}-\gamma_{B}}{2} \frac{l}{\pi \sqrt{x(l-x)}}, \\
& \eta_{B}(x)=\frac{\gamma_{A}+\gamma_{B}}{2}+\frac{\gamma_{B}-\gamma_{A}}{2} \frac{l}{\pi \sqrt{x(l-x)}},
\end{aligned}
$$
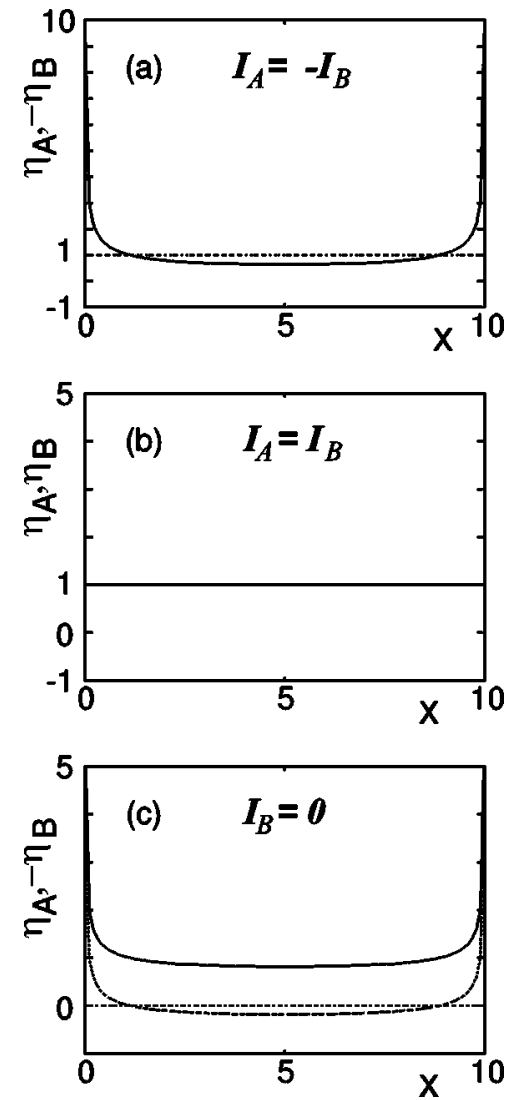

FIG. 3. The current distributions $\eta_{A}$ and $\eta_{B}$, calculated from Eqs. (26) for a junction of length $l=10$, are plotted assuming $\left|\gamma_{A, B}\right|=1$ (singularities at the edges have been eliminated). (a) The nonuniformity is the largest, the two distributions mirror each other; for sake of comparison, the uniform distribution $\left|\eta_{A, B}=1\right|$ is also plotted. (b) The currents in the two juctions are equal and uniform. (c) The nonuniform current in $A$ (continuous line) induces a nonuniform current in $B$ (dashed line); the net current in $B$, however, is null.

and the other symbols have the same meaning of the uniform current model. The above equations could also be derived using the approach of Ref. 1.

Note that here we have maximum nonuniformity for equal and opposite physical bias currents $\left(I_{A}=-I_{B}\right)$. Perfect uniformity is obtained for equal bias currents $\left(I_{A}=I_{B}\right)$, but we remark that this is a result of the assumption of uniform current distribution made for the outer electrodes. How good it is this approximation can be established only by comparison with the experimental results, that we will present in the next section. Figure 3 shows a sketch of distribution of the normalized current densities $\eta_{A}(x)$ and $\eta_{B}(x)$ for the cases $I_{A}=-I_{B}$ and $I_{A}=I_{B}$. For other cases the current distribution is somewhere in between Figs. 3(a) and 3(b). A particular interesting case is that for which only one junction is biased, e.g., $I_{B}=0$. In this case [Fig. 3(c)], there is a nonzero current distribution in junction $B$, such that current flows in two opposite directions. However, net current flow through junction B is, consistently, zero, as can be easily checked calculating $\int_{0}^{l} \eta_{B}(x)$ from Eq. (26) for $\gamma_{B}=0$. From the understanding of the behavior of two junction stacks with the geometry of Fig. 2, it is easy to generalize to more junctions with different variations of the geometry of Fig. 2. 


\section{EXPERIMENTAL RESULTS}

\section{A. The samples}

We have fabricated and tested $\mathrm{Nb} / \mathrm{AlO}_{x} / \mathrm{Nb} / \mathrm{AlO}_{x} / \mathrm{Nb}$ stacks with electrodes patterned in the double overlap geometry shown in Fig. 2. In all of tested samples the intermediate electrode was thinner than the London penetration depth $\lambda_{L}$ while the external electrodes were quite larger than $\lambda_{L}$. Due to the fabrication process, $\lambda_{L}$ in our $\mathrm{Nb}$ films is rather large, about $100 \mathrm{~nm}$. The physical dimensions of the junctions in the stacks were $L \times W=(600 \times 20) \mu \mathrm{m}^{2}$ and a typical Josephson penetration depth $\lambda_{J}$ was greater than $40 \mu \mathrm{m}$, so that we have long and narrow junctions. In the two representative samples on which we report here, the magnetic coupling constant was $\varepsilon=-0.56$ and $\varepsilon=-0.89$, respectively. These values are calculated by Eq. (7) and agree with those extimated by the procedure based on the splitting of voltage spacing of Fiske steps. ${ }^{14}$ In the stack with $\varepsilon=$ -0.56 the two junctions have very similar critical current densities (evaluated by the current rise in the $I-V$ characteristic at the gap sum voltage) $J_{A}=76 \mathrm{~A} / \mathrm{cm}^{2}, J_{B}$ $=80 \mathrm{~A} / \mathrm{cm}^{2}$, and normalized length $l_{A} \approx l_{B}=13$. In the other stack $(\varepsilon=-0.89)$ the critical current densities are slightly different, $J_{A}=66 \mathrm{~A} / \mathrm{cm}^{2}, J_{B}=60 \mathrm{~A} / \mathrm{cm}^{2}$, and the normalized lengths are $l_{A} \sim l_{B}=10$.

\section{B. Measurements}

The experiments were performed at $4.2 \mathrm{~K}$ with a cryoperm shielded sample holder and in zero applied magnetic field. We carefully avoided trapped flux and checked that there was no crosstalk between the biasing circuits.

The measurements concern how the stability of the zero voltage state of one junction is influenced by the state of the other junction in the same stack. In other words we have measured the critical current of one junction as a function of the bias current in the other junction, in both possible voltage configurations for the second junction: zero voltage state or McCumber state. Typical experimental results are summarized in Fig. 4. Figure 4(a) shows the stability boundary of the zero voltage state in the stack, i.e., in the region between the two curves in the plane $I_{c A}-I_{c B}$ is $V_{A}=V_{B}=0$. To obtain this stability boundary we start with both junctions in the zero voltage state, then we fix the bias of one junction and we change the current in the other junction until it switches to a resistive state. Equivalently, we can start with both junctions in the zero voltage state (at $I_{A}=I_{B}=0$ ) and then change both bias currents simultaneously until one junction undergoes a transition to the dynamical state. In Fig. 4(b) we show the dependence of the positive and negative critical current of junction $A$ as a function of the current in junction $B$ biased on the McCumber curve. Similar results are obtained inverting the role of the junctions. We notice absence of experimental points around $I_{B}=0$. This is because for small values of current in junction $B$ we are in the instability region of the McCumber curve. Finally, we note that in the range of currents in $B$ where a comparison is possible, the critical current in junction $A$ depends on the voltage state of the junction $B$, as shown in an expanded scale in Fig. 4(c).
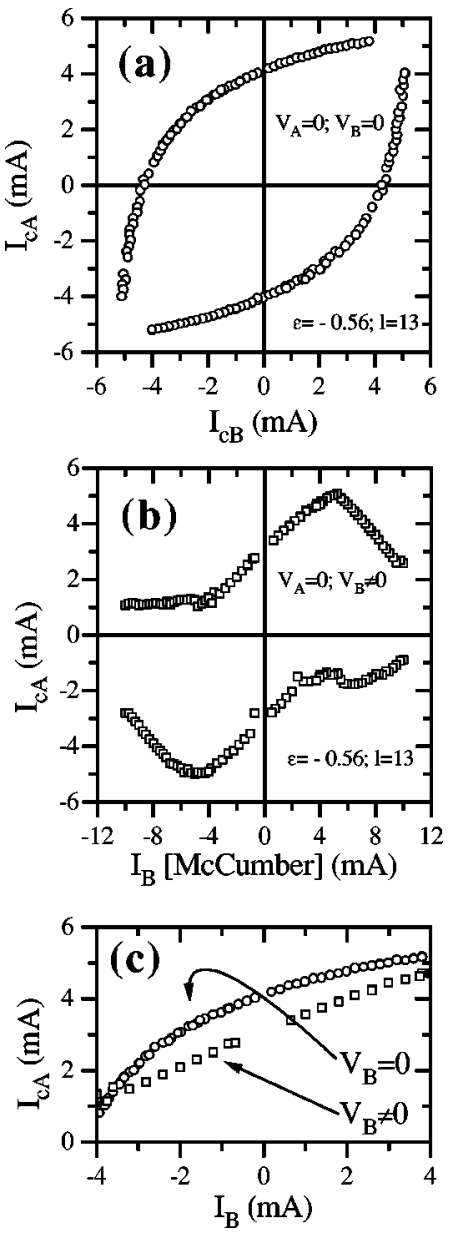

FIG. 4. (a) Stability boundary of the zero voltage state in the stack. (b) Critical current of junction $A$ versus current in junction $B$ on the McCumber curve. (c) Critical current of junction $A$ versus current in junction $B$ polarized in the zero voltage state (circles) or in the McCumber state (squares).

The results shown in Fig. 4 are obtained without a ground plane. Stability boundaries of the zero voltage state in the same stack with and without a ground plane are compared in Fig. 5(a). From this figure is evident that the influence of a ground plane is relevant only in the region around the line $I_{A}=-I_{B}$. We remark that the ground plane was spaced about $1 \mathrm{~mm}$, the thickness of the substrate, from the stack, so its function was quite weak.

Nevertheless, Fig. 5(a) suggests that some nonuniformity in current distribution is working, especially when $I_{A} \approx$ $-I_{B}$. Nonuniformity should create some appreciable second spatial derivative of the phases and consequently an increasing of (static) interaction between junctions for increasing inductive coupling constant $\varepsilon$. In Fig. 5(b) is shown the stability range of the zero voltage state for stacks with two different $\varepsilon$. To allow comparison, in this plot we have normalized, in each of the stacks, the currents to the value of the critical current of junction $A$ for junction $B$ unbiased. Besides the deformation of the stability range, due to the difference between critical currents of the junctions in the stack with $\varepsilon=-0.89$, we note that increasing of the inductive coupling effectively reduces the stability range (i.e., increases static interaction between junctions), again principally around $I_{A}=-I_{B}$. 

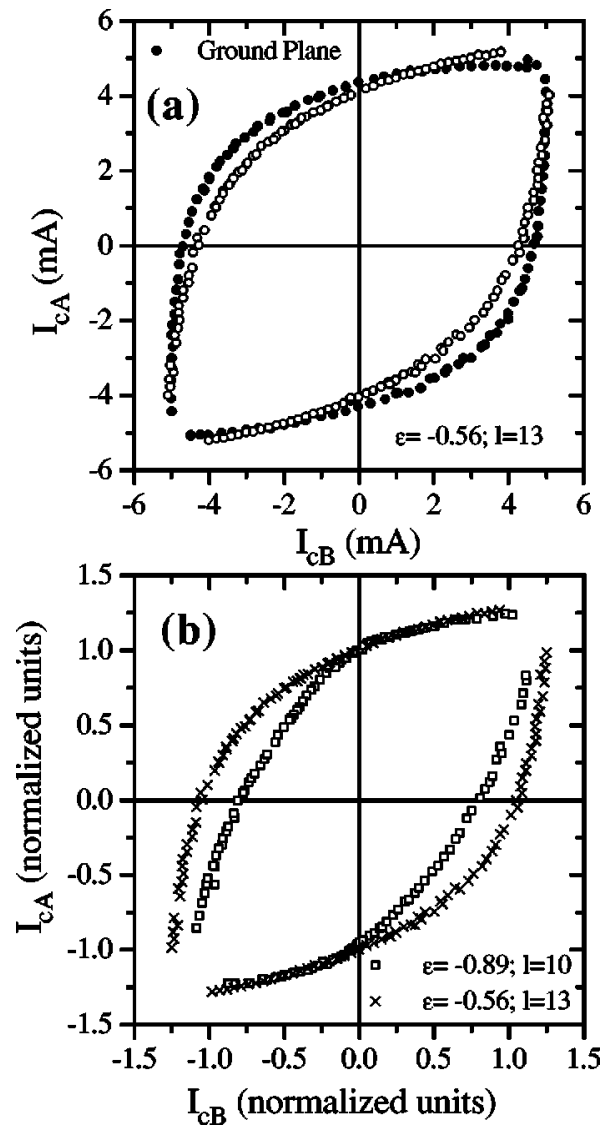

FIG. 5. (a) Stability boundary of the zero voltage state without a ground plane (open circles) and its modification caused by a remote ground plane (solid circles). (b) Range of existence of the zero voltage state in a stack with $\varepsilon=-0.56$ (crosses) and in a stack with $\varepsilon=-0.89$ (open squares). In both curves, the currents are normalized to $I_{c A}\left(I_{B}=0\right)$.

\section{Discussion}

In the uniform model Eqs. (20) interaction between the junctions takes place only when $\varphi_{x x}$ or $\psi_{x x}$ are different from zero. If, as in our experiments, we have not trapped flux in the films, we have not applied magnetic field and we are not in some solitonic zero-field step (ZFS) regime, $\varphi_{x x}$ and $\psi_{x x}$ should be zero and consequently the boundary of stability in Fig. 4(a) should be a square and the dependence in Fig. 4(b) a constant. So, the uniform model Eqs. (20) cannot explain our experimental results.

Below we consider several possible mechanisms that may modify the predictions of the uniform junction model. The interaction in the zero voltage state looks similar to earlier experimental observations ${ }^{15}$ in closely coupled microbridges that was explained in terms of current-induced orderparameter depression in the region between the bridges. ${ }^{15,16}$ Nevertheless, in Ref. 15 the current in a weak link was always depressing the critical current in the other, while in our results the depression is obtained only for opposed currents, in agreement with results of Ref. 17 that were explained with a Cooper pair coupling between the outer electrodes of the two weak links. ${ }^{17,18}$ Both the quoted experimental results were obtained near the critical temperature of the superconductors, to have a coherence length quite larger than the spacing between weak links. This is not the case for most of our stacks in which we observe interaction. However, we notice that our stacks are double-barrier tunnel devices, qualitatively different from the coupled microbridges where the above-mentioned interaction was investigated. In our case, the Cooper pair tunneling between outer electrodes (if any) should be of the order of the single electron resonant tunneling ${ }^{19}$ between the same electrodes. Such a tunneling should be appreciable in the limit of extremely thin (i.e., few nanometers) intermediate electrode. Due to the macroscopic thickness of our intermediate electrodes, we can rule out the presence of such a coupling in our experimental results. Also, we notice that induced gap suppression is too small, lower than $2 \%$ for typical used current values, to account for the observed large variations of critical currents. This, together with the too small quasiparticle curve modification, also weakens the hypotheses that some nonequilibrium process of quasiparticle injection is causing the results of Fig. 4.

The results of Fig. 5 suggest that we should look for the predominant mechanism in some spatial nonuniformity. The results in Fig. 5(a) rule out the possibility of a spatially dependent critical current density. In fact, such a critical current density should be caused by a shaped barrier thickness, that should not depend on a ground plane action. Absence of defects or pinning centers in the barriers has been demonstated by dedicated measurements (diffraction patterns in magnetic field) performed on single junctions fabricated with the same fabrication process as the stacks. Finally, we have carefully avoided misalignments of the electrodes forming the junctions in the stack to reduce in-line current components.

From all this, we conclude that the most effective mechanism is the nonuniformity and this nonuniformity is due to the bias currents. So we will describe our experimental results with the model Eqs. (25).

\section{Numerical simulations}

We have integrated the model Eqs. (25) without applied magnetic field:

$$
\begin{gathered}
\varphi_{x x}-\varphi_{t t}=\sin (\varphi)+\alpha \varphi_{t}+\varepsilon \psi_{x x}-\eta_{A}(x), \\
\psi_{x x}-\psi_{t t}=\sin (\psi)+\alpha \psi_{t}+\varepsilon \varphi_{x x}-\eta_{B}(x), \\
\varphi_{x}(0)=\varphi_{x}(l)=0, \\
\psi_{x}(0)=\psi_{x}(l)=0 .
\end{gathered}
$$

To remove singularities at edges in the forcing terms $\eta_{A}(x)$ and $\eta_{B}(x)$, we have used the relation Eq. (22). Another possibility is to choose values at the edges such that the physical relation (charge conservation)

$$
\gamma_{A, B}=\frac{1}{l} \int_{0}^{l} \eta_{A, B}(x) d x
$$

is numerically satisfied, as it is analytically. Numerically, we have found no significant differences between these two methods.

The values of the parameters in the simulation are chosen equal to the experimental ones, so that a direct comparison can be made. Experimental and numerical results for $\varepsilon=$ -0.56 are shown in Fig. 6. Globally, we found the agree- 

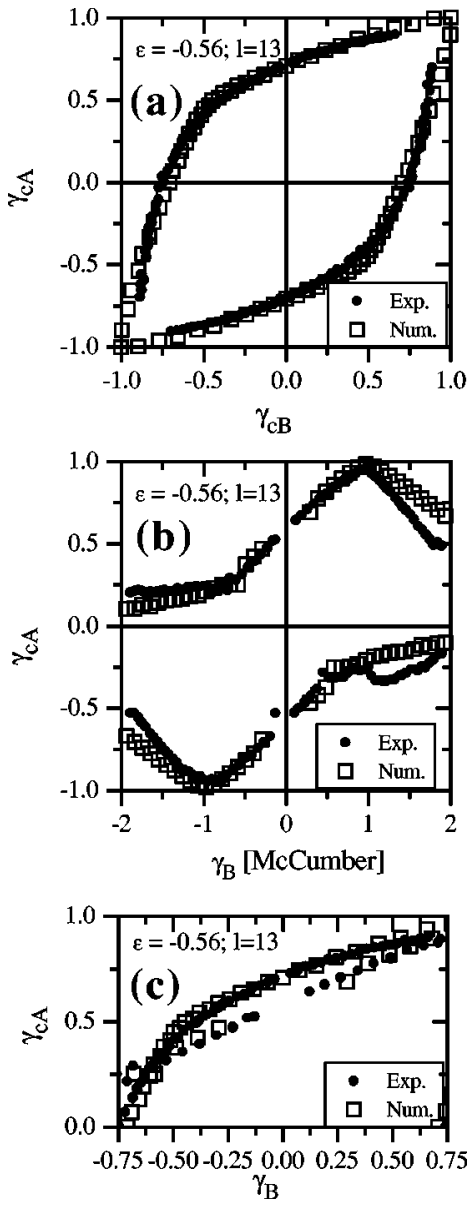

FIG. 6. Experimental results (solid circles) of Fig. 4 compared with the numerical results (open squares) of the nonuniform model. Both in the experiment and in the simulation was $\varepsilon=-0.56, l_{A}$ $=l_{B}=13, \alpha=0.1, \quad \eta=0$.

ment quite satisfactory. In Fig. 6(b) a significant deviation is evident for $\left|\gamma_{B}\right|>1.3$ where mechanisms other than nonuniform current distribution, possibly quasiparticle injection, play a role. A small deviation is evident in Fig. 6(a), around the line $\gamma_{A}=\gamma_{B}$ where the experimental points seem not to point to the values $\gamma_{C A}=\gamma_{C B}= \pm 1$. This means, by considerations of the previous section, that bias current distribution in the outer electrodes is not perfectly uniform. Nevertheless, we found that the deviation is small enough to exempt us from a further correction to the model.

In Fig. 7, numerical and experimental results are compared for the stack with $\varepsilon=-0.89$. In the simulations, the relatively small difference between critical current densities of the junctions has been accounted for introducing only one parameter in the model. In fact, if the difference is not so large, we can account for it by introducing only the ratio $j$ $=J_{B} / J_{A}$ to multiply the $\sin (\psi)$ term in Eqs. (27). The result in Fig. 7, obtained with $j=0.85$, shows that this is enough to reproduce quite well the deformation of the stability boundary of the zero voltage state observed when the critical currents are different.

In Fig. 8 we show the spatial phase distribution for some selected states of the stack. In Fig. 8(a), the phase of junctions $A$ and $B$ are recorded just before and just after junction $A$ undergoes the transition to the dynamical state, while junction $B$ is unbiased. Here we can note that the transition of

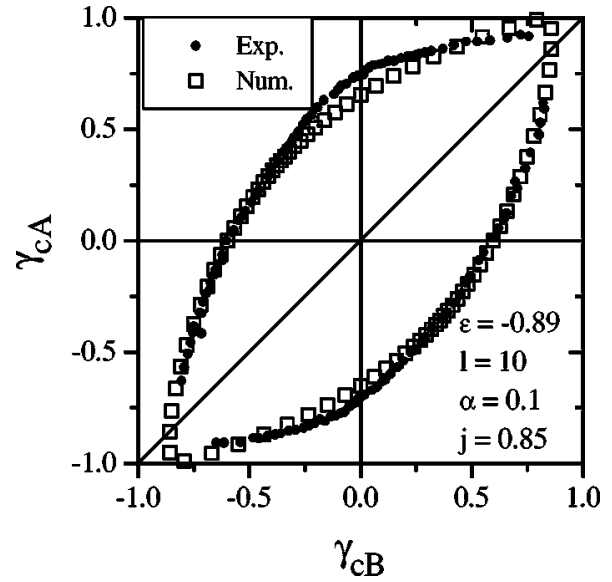

FIG. 7. Experimental results for the stack with slightly different critical current densities $\left(J_{B} / J_{A}=0.9\right)$ compared with the numerical results. In the simulation was $l_{A}=l_{B}=10$ and $j=J_{B} / J_{A}=0.85$.

junction $A$ is caused by the formation of a fluxon and an antifluxon at the opposite edges of the junction, and that the phase of the unbiased junction is spatially modulated mirroring (because of the inductive coupling) the phase of the biased junction. In Figs. 8(b) and 8(c) is shown the phase distribution of junction $A$ (in the zero voltage state), while $B$ is
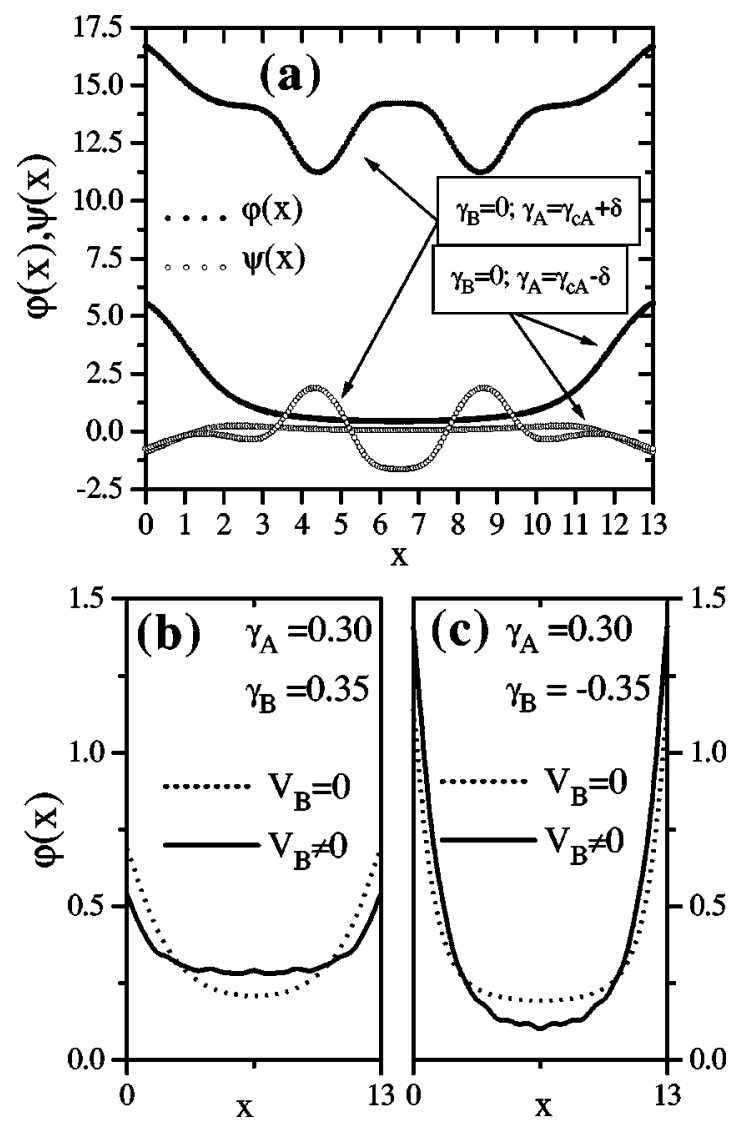

FIG. 8. (a) Phase of junction $A[\varphi(x)]$ and junction $B[\psi(x)]$ for junction $B$ unbiased and junction $A$ biased with a current slightly lesser or slightly greater than its critical current $\gamma_{c A}$. (b) Phase of junction $A$ in the zero voltage for junction $B$ also in the zero voltage state (dashed line) or on the McCumber curve (solid line). (c) Same as that in (b), but here the bias currents have opposite polarities. 
biased at $V_{B}=0$ or $V_{B} \neq 0$, in two configurations of relative current polarities. Here, beside the different degree of nonuniformity caused by the term $\eta_{A}(x)$, we can notice the small modulation induced on the phase distribution when $V_{B} \neq 0$. This modulation is observed also in the phase distribution of junction $B$ and results from possibile excitation of self-resonances in the $I-V$ characteristic.

\section{CONCLUSIONS}

We have discussed the results obtained in the stationary state of stacked long Josephson junctions, but, obviously, the model can account for deviation from the ideal uniform model also in the dynamical state. However, because of the very slow dependence from the junction length, we expect that the interaction due to the bias currents should not be negligible in three-terminal devices with double overlap geometry also in the limit of small junctions. The geometry discussed here is a special one, but the results presented in this paper indicate that the geometrical factors should not be overlooked for a full description of the three-terminal devices.

\section{ACKNOWLEDGMENTS}

During preparation of this paper, we could not forget the interest and the stimulus received by our late Professor R. D. Parmentier. Fruitful discussions with M. R. Samuelsen and preliminary numerical work of G. Filatrella are also acknowledged.
${ }^{1}$ S. Sakai, P. Bodin, and N. F. Pedersen, J. Appl. Phys. 73, 2411 (1993).

${ }^{2}$ A. Barone and G. Paternó, Physics and Applications of the Josephson Effect (Wiley, New York, 1982).

${ }^{3}$ G. Carapella, G. Costabile, A. Petraglia, N. F. Pedersen, and J. Mygind, Appl. Phys. Lett. 69, 1300 (1996).

${ }^{4}$ G. Carapella and G. Costabile, Appl. Phys. Lett. 72, 377 (1998).

${ }^{5}$ J. C. Eilbeck, P. S. Lomdahl, O. H. Olsen, and M. R. Samuelsen, J. Appl. Phys. 57, 861 (1985).

${ }^{6}$ E. H. Rhoderick and E. Wilson, Nature (London) 194, 1167 (1962).

${ }^{7}$ V. L. Newhouse, J. W. Bremer, and H. H. Eduards, Proc. IRE 48, 1395 (1960).

${ }^{8}$ O. H. Olsen and M. R. Samuelsen, J. Appl. Phys. 54, 6522 (1983).

${ }^{9}$ M. R. Samuelsen and S. A. Vasenko, LT-17 (Contributed Papers), edited by U. Eckern et al. (Elsevier Science, Amsterdam, 1984).

${ }^{10}$ N. F. Pedersen and D. Welner, Phys. Rev. B 29, 2551 (1984).
${ }^{11}$ A. Barone, F. Esposito, K. K. Likharev, V. K. Semenov, B. N. Todorov, and R. Vaglio, J. Appl. Phys. 53, 5802 (1982).

${ }^{12}$ K. K. Likharev, Dynamics of Josephson Junctions and Circuits (Gordon and Breach, New York, 1986).

${ }^{13}$ T. Van Duzer and C. W. Turner, Principles of Superconductive Devices and Circuits (Elsevier North-Holland, New York, 1981).

${ }^{14}$ S. Sakai, A. V. Ustinov, H. Kohlstedt, A. Petraglia, and N. F. Pedersen, Phys. Rev. B 50, 12905 (1994).

${ }^{15}$ D. W. Jillie, J. E. Lukens, and Y. H. Kao, Phys. Rev. Lett. 38, 915 (1977).

${ }^{16}$ Y. S. Way, K. S. Hsu, and Y. H. Kao, Phys. Rev. Lett. 39, 1684 (1977).

${ }^{17}$ H. J. T. Smith and M. Dion, Phys. Rev. B 42, 206 (1990).

${ }^{18}$ G. Carapella, G. Costabile, R. De Luca, S. Pace, A. Polcari, and C. Soriano, Physica C 259, 349 (1996).

${ }^{19}$ Tunneling Phenomena in Solids, edited by E. Burstein and S. Lundqvist (Plenum, New York, 1969). 